\title{
多深度特征增强与顶层信息引导的边缘检测网络
}

\author{
朱威，岑宽，徐希舟，何德峰 \\ (浙江工业大学信息工程学院 杭州 310023) \\ (weizhu@zjut.edu.cn)
}

\begin{abstract}
摘 要：针对现有边缘检测网络在复杂自然场景下的检测结果仍存在边缘缺失、噪声过多等问题，提出多深度特征 增强与顶层信息引导的边缘检测网络. 首先, 采用 UNet++作为主干网络提取不同深度的特征，并通过特征叠加使不 同尺度的边缘更加完整; 然后, 在每个卷积层的侧输出后添加特征增强模块, 通过引人空洞卷积增大感受野, 增强多尺 度信息; 最后，设计顶层信息引导模块，通过在跳跃连接中引人高层的语义特征增强边缘检测效果. 实验结果表明，在 BSDS500, NYUDv2 和 Multicue 这 3 个数据集上进行训练均取得了较好的效果, 其中, BSDS500 数据集上的 ODS, OIS 和 AP 指标分别达到了 $0.821,0.839$ 和 0.869 , 整体上高于现有边缘检测网络, 且噪声少, 主观效果也更接近真值.
\end{abstract}

关键词：边缘检测网络; UNet++; 多深度特征; 特征增强; 顶层信息引导

中图法分类号: TP391.41 DOI: 10.3724/SP.J.1089.2021.18752

\section{Edge Detection Network with Multi-Depth Feature Enhancement and Top-Level Information Guidance}

Zhu Wei, Cen Kuan, Xu Xizhou, and He Defeng

(College of Information Engineering, Zhejiang University of Technology, Hangzhou 310023)

\begin{abstract}
The existing edge detection networks still have problems such as missing edges and excessive noise in complex natural scenes. Therefore, an edge detection network with multi-depth feature enhancement and top-level information guidance is proposed. First, UNet++ is used as the backbone network to extract features of different depths, and the edges of different scales are made more complete by feature superposition. Then, a feature enhancement module is added after the side output of each convolution layer to increase the receptive field and enhance the multi-scale information by introducing the dilated convolution. Finally, a top-level information guidance module is designed to enhance the edge detection effect by introducing top-level semantic features into jump connection. The experimental results show that training on the three datasets of BSDS500, NYUDv2 and Multicue has achieved good results. On the BSDS500 dataset, the ODS, OIS and AP of this network reach 0.821, 0.839 and 0.869 respectively, which is generally higher than the existing edge detection networks. Moreover, the result has less noise and the subjective effect is closer to the ground truth.
\end{abstract}

Key words: edge detection network; UNet++; multi-depth feature; feature enhancement; top-level information guidance

收稿日期：2020-11-02；修回日期：2021-07-11. 基金项目：浙江省自然科学基金(Y21F010051); 国家自然科学基金(61773345); 汽车仿真与控制国家重点实验室开放基金(20171103). 朱威(1982-), 男, 博士, 副教授, 硕士生导师, 主要研究方向为模式识别与智 能系统; 岑宽 (1997一), 男, 硕士研究生, 主要研究方向为智能视觉处理; 徐希舟(1997一), 男, 硕士研究生, 主要研究方向为智能视 觉处理; 何德峰(1979-), 男, 博士, 教授, 博士生导师, 主要研究方向为智能驾驶与安全控制. 
边缘检测旨在感知并识别自然图像中视觉显 著的边缘和边界，是近年来计算机视觉领域的一 项重要的任务. 图像的分析理解过程中常常会用 到边缘检测技术，如图像分割 ${ }^{[1]}$ 、目标检测 ${ }^{[2]}$ 和目 标识别 ${ }^{[3]}$ 等. 此外, 自动驾驶和智能图像压缩等应 用也需要用到高质量边缘的检测.

传统的边缘检测器主要利用图像的强度和颜 色梯度突出边缘, 如 Sobel 算子 ${ }^{[4]}$ 在灰度图像上通 过滑动 $3 \times 3$ 的滤波器获取对边缘像素响应的梯度 图像. Canny 算子 ${ }^{[5]}$ 进一步通过高斯滤波进行降噪 处理，并采用非最大抑制(non-maximum suppression, NMS $)^{[6]}$ 提取较细的边缘轮廓. 虽然这些传统 的边缘检测器在计算机视觉领域仍在广泛应用, 但是也有着明显的局限性. 在比较复杂的自然场 景图像中，仅利用低级特征难以自适应地获得多 尺度的边缘。

为了解决传统边缘检测器的缺陷, 研究人员 逐渐使用一些低级特征手动设计边缘检测器, 并 使用机器学习的方法对图像边缘像素进行分类. Konishi 等 ${ }^{[7]}$ 通过学习对应于 2 组边缘滤波器的响 应概率分布，提出了一种基于数据驱动的边缘检 测方法. Martin 等 ${ }^{[8]}$ 发现了添加纹理渐变可以显著 提高边缘效果. 而后, 研究人员又探索了基于学习 的边缘检测方法. Dollár 等 ${ }^{[6]}$ 提出了一种数据驱动、 有监督的边缘检测器，将检测问题归结为一个稠 密的二值标记问题，使特征集中在局部区域. 然 而, 这些方法在表达高水平边缘特征时仍存在着 明显的问题.

近年来，随着人工智能的快速发展，基于深度 学习的边缘检测方法取得了巨大的进步. Ganin 等 ${ }^{[9]}$ 将最近邻搜索与卷积神经网络 (convolutional neural networks, CNN)相结合, 提出了 $N^{4}$-Fields, 通过匹配图像块得到边缘特征。在上述工作基础 上, Bertasius 等 ${ }^{[10]}$ 利用 $\mathrm{CNN}$ 生成候选轮廓点的特 征. Xie 等 ${ }^{[11]}$ 设计了一种简单高效的整体嵌套边缘 检测器，该方法对图像全局进行计算，通过端到端 的方式收集每个卷积层的特征. Liu 等 ${ }^{[12]}$ 提出了一 种更为精确的边缘检测器, 通过加强所有卷积层 的特征融合，学习更丰富的多尺度边缘特征. 此 后， $\mathrm{He}$ 等 $^{[13]}$ 在其基础上做出了改进，通过增强每 层的特征输出并采用双向级联网络进一步增强多 尺度的特征融合，显著提升了检测效果.

虽然基于深度学习的边缘检测网络取得了较 好的成果, 但其仍存在很多问题值得解决. 一幅图 像的边缘由对象级的边界轮廓和局部细节组成.
不同人对边界轮廓的感知大致相同，而局部细节 上有着明显的差异. 为了产生更加细致的多尺度 特征, 以弥补细节上的边缘信息, 一些研究人员采 用 VGG16 ${ }^{[14]}, \operatorname{ResNet50} 0^{[15]}$ 和 ResNet101 ${ }^{[15]}$ 等的特 征提取网络作为边缘检测的主干网络. 其中, ResNet50 的特征提取网络卷积层参数量比 VGG16 多很多，但更多的参数对整体边缘检测效果的提 升比较有限. 对于需要较高运算速度的边缘检测, 是否可以使用浅层网络获得更好的性能? 另外, 已有研究者选用 U-Net ${ }^{[16]}$ 结构提取图像特征. 尽管 从 U-Net 的原理可知, 通过对输人图像的降采样和 上采样操作可以大大提高图像分割的精度，然而， U-Net 仅在第 4 层深度返回, 是否可以改变 U 型网 络的返回深度进一步提高网络性能?

为了解决上述问题, 本文提出了多深度特征增 强和顶层信息引导的边缘检测网络. 该网络采用 $\mathrm{UNet}++^{[17]}$ 作为主干网络, 用于提取多深度的图像 边缘特征. 在降采样过程设计了特征增强模块, 以 增强跳跃连接时的侧边图像精度. 在上采样过程利 用 UNet ++ 融合不同深度的特征, 并在横向连接时 通过设置顶层信息引导模块, 增强边缘的高水平语 义信息. 最后设计整合模块, 以减少过度上采样带 来的混叠效应, 优化最终输出的边缘检测图像.

\section{1 本文网络}

\section{1 网络总体结构}

图 1 所示本文网络总体结构以 VGG16 作为基 础网络, 并搭配自下而上的 U 型网络结构. 首先, 删 除了 VGG16 的最后 3 个全连接层和 1 个池化层, 再 将剩余的 13 个卷积层分成 5 个块(block1 block5), 每块后跟随一个池化层, 以逐步扩大下一个块中 的感受野. 然后, 为了增强网络侧输出的多尺度特 征，在每个卷积层后都跟随了一个特征增强模块 (feature enhancement module, FEM), 该模块由空 洞卷积组成, 大幅度减少了网络参数, 有效地避免 了几余计算. 接着, 本文没有使用传统的 U-Net, 而是选择了性能更强的 UNet++结构, 该结构使浅 层和深层的特征都得到更充分的利用. 最后，在 $\mathrm{UNet}++$ 的上采样过程中, 本文设计了顶层信息引 导模块 (top-level information guidance module, TGM), 辅助网络捕获图像中边缘的整体轮廓; 同 时该模块中的整合结构又进一步降低了上采样导 致的混叠效应, 提升了边缘检测的效果. 图 1 上半 部分为网络的整体结构, 参照了 UNet++结构, 下 
半部分第 1 个红色方框给出了 block1 block5 的具 体划分和连接, 每个 block 中卷积层的侧边都连接 了 FEM 用于增强从低级特征到高级语义特征的多 尺度信息, 并将结果融合后作为每个 block 的侧输 出. temp 模块为中间特征变量用于上采样时融合 来自不同层次 $\mathrm{U}$ 型网络的边缘特征, 其中包含了 不同数量的长短连接; res 模块为 UNet++中 1 4 层
的边缘特征输出, layer_1 layer_4 共同组成最终的 图像边缘. 图 1 下半部分第 2 个红色方框给出了 UNet++中第 4 层网络的具体实现细节, 其中 TGM 用于高级信息的全局引导, 以弥补后续上采样过 程中被稀释的顶层语义信息，在每次上采样并融 合后都需要连接整合模块 $E$, 该模块用于辅助特征 信息的融合和增强.

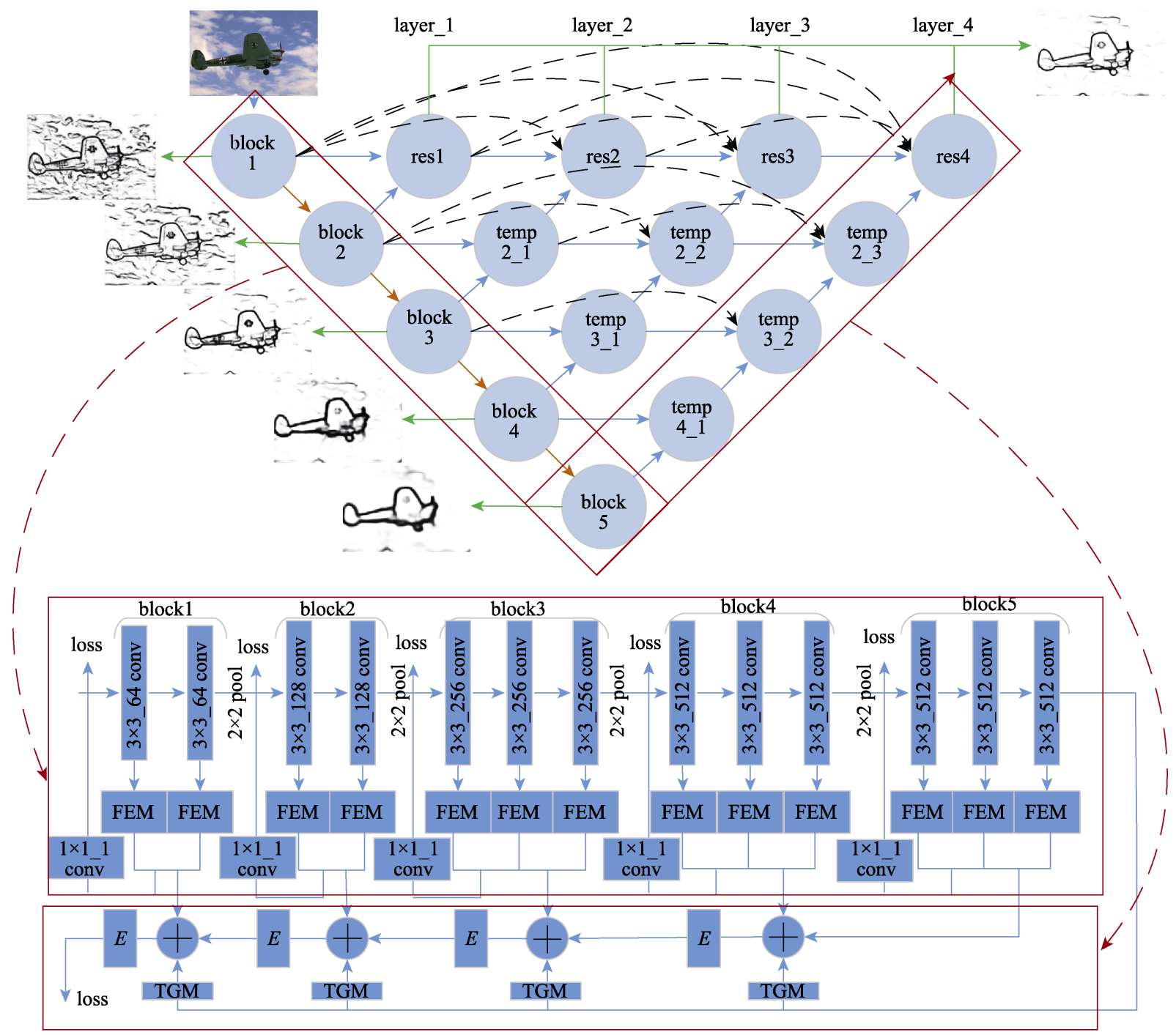

图 1 本文网络结构

\section{2 主干网络结构}

U-Net 框架用于医学领域图像的分割，由编码 器、解码器和跳跃连接 3 部分组成. 编码器通过不 断地进行卷积和池化操作分解多深度的图像特征, 并提取出上下文信息; 解码器通过上采样传递并 还原各层的细节特征; 跳跃连接则是作为编码器 和解码器的连接通道, 强化信息的定位和提取, 使 图像精确分割.

UNet++是在 U-Net 的基础上针对跳跃连接进
行了改进. 与原来 4 层的 U-Net 相比, UNet++将剩 余 1 3 层的 $U$ 型结构全部连接在一起, 这种长连接 和短连接互补的方式使编码器相邻等级的语义特 征水平更为接近. 当接收到的编码器特征映射和 相应的解码器特征映射在语义上更相似时, 网络 优化能力会大大提升.

UNet ++ 的结构如图 1 上半部分所示, 该网络 一共由 1 4 层不同深度的 U-Net 组成. 不同网络层 之间通过将解码器上采样后的特征与下一层相对 
应的模块特征融合形成一个完整的 $U$ 型网络. 每 个模块的输出为

$$
x^{i, j}=\left\{\begin{array}{ll}
H\left(x^{i-1, j}\right), & j=0 \\
H\left(\left[\left[x^{i, k}\right]_{k=0}^{j-1}, U\left(x^{i+1, j-1}\right)\right]\right), & j>0
\end{array} .\right.
$$

其中, $x^{i, j}$ 表示 $x$ 特征提取模块的输出结果; $i$ 表 示编码器下采样层的索引; $j$ 表示相同层数模块 的序号， $j=0$ 时表示节点只接收来自编码器前一 层的输人, $j>0$ 时表示节点接收 $j+1$ 个跳跃连接 输人; $H(\cdot)$ 表示特征提取模块的卷积操作以及激 活函数; $U(\cdot)$ 表示上采样层; [.] 表示特征连接层.

本文将 UNet++的 block1 block5 作为主干网 络, 其中每个 block 在进行特征增强后都添加一个 $1 \times 1 \_1$ 的卷积层, 在上采样至原图大小后对图像 进行监督，该设计可以提升后续编解码过程中图 像特征的准确性. temp 模块作为中间变量, 用作特 征信息的过滤、融合和传递，该模块由 2 个 $3 \times 3$ 的 卷积层和 ReLU 激活函数组成, 在每次卷积操作后 会进行数据归一化处理, 防止数据过大导致网络 性能不稳定. 由于不同层中间对应的跳跃连接数 量不同, 卷积后需要将拼接后的特征图维度降为 该层的原始数量. 另外, 本文网络在最外层的 temp 模块的跳跃连接部分添加了 TGM.res 模块作 为最后的输出层, 本文将 res1 res4 这 4 个输出结 果分别进行监督并计算其损失, 最后将所有输出 结果融合作为最终的边缘图. 在实验中, UNet++感 受野更丰富，能抓取不同层次的特征，并通过特征 叠加的方式使不同尺度的边缘更加完整.

\subsection{FEM}

由于选用了 VGG16 的特征提取网络作为主干 网络，该结构仅由多个卷积层堆叠而成. 如果仅对 侧输出进行简单的卷积操作就作为每个 block 的边 缘特征，空间和特征维度的信息会被局部感受野 所限制，无法提取图像的全局特征，网络表达能力 会有一定的下降. 考虑单一尺度的特征往往无法 检测出完整的边缘信息, 本文受到 Chen 等 ${ }^{[18]}$ 提出 的空洞卷积模型的启发，设计了边缘特征增强结 构, 以丰富边缘的多尺度特征. 与传统卷积相比, 空洞卷积使用了过滤器大小相同的卷积核, 通过 改变膨胀系数大小增大感受野, 从而增强边缘的 多尺度特征; 同时该结构有效地降低了模型的参 数量, 避免了几余计算. 空洞卷积核尺寸与膨胀系 数关系为 $K_{\text {size }}=(r-1) \times\left(k_{\text {size }}-1\right)+k_{\text {size }}$. 其中, $K_{\text {size }}$ 为空洞卷积核的大小; $r$ 为膨胀系数, 表示采样输
人特征映射的步长; $k_{\mathrm{size}}$ 为普通卷积核大小. 当 $r=1$ 时, $K_{\text {size }}$ 即为普通卷积核.

FEM 结构如图 2 所示. 首先考虑该模块紧接 每个卷积层的侧输出, 不同卷积层的输出通道数 不同，在特征增强前先让中间特征图经过 $3 \times 3$ _ 32 的卷积核和激活函数, 统一特征图的维数. 接着经 过 3 组不同膨胀系数的卷积核, 经过实验, 本文将 膨胀系数 $D$ 设置为 $4,8,12$. 最后将 3 组空洞卷积 的结果与原特征图相加融合, 经过一个 $1 \times 1 \_21$ 的 卷积降低特征图的维度, 得到每个 block 的最终侧 输出, block1 block5 的侧输出用于后续网络的横 向连接和跳跃连接. 与直接输出相比, 特征增强后 的边缘效果更佳.

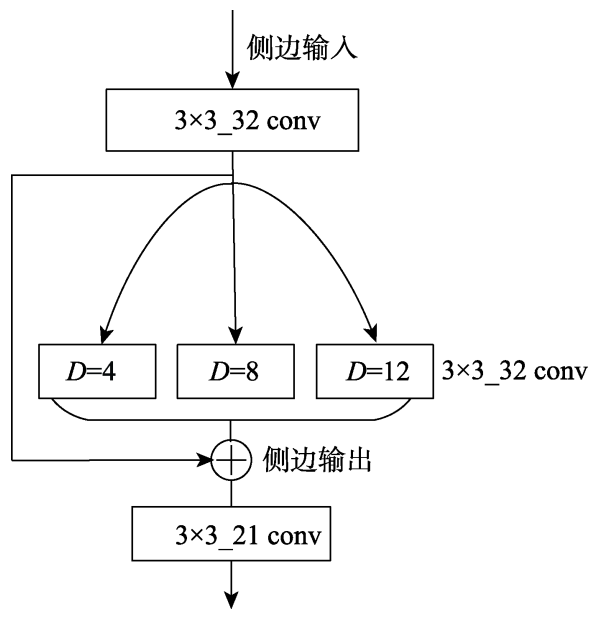

图 2 FEM 具体网络结构

\subsection{TGM}

在一些目标分类和检测网络中, 研究者常通 过融合不同尺度的特征提高图像分割的精度. 低 层次的边缘特征经过的卷积较少, 因此分辨率高, 有着较多的细节信息, 但是噪声多, 语义性也低. 高层次的特征经过大量的滤波, 具有更强的语义 特征，但是分辨率低，且细节信息被大量丢失。然 而, 在与低层次特征融合时, 高层次的特征往往会 被覆盖，导致部分信息的缺失. 因此，如何高效地 融合不同层次的特征，成为改善边缘检测网络性 能的一大要点.

为减少高水平语义信息的丢失, 本文在网络 中设计了信息引导网络结构, 以引导顶层语义信 息, 如图 3 所示. 该结构由引导模块和整合模块 2 部分组成. 引导模块用于顶层语义信息的提取和 分流，整体模块与金字塔池化模块相似，不同的是 传统的金字塔池化模块是嵌人在网络架构的某层 中, 本文将该模块作为一个独立的模块参与每层 
的信息引导以补充被稀释的高层语义信息. 首先, 获取顶层的特征图，并采用 3 种不同的金字塔尺度 进行自适应平均池化操作，尺度大小分别为 $1 \times 1$, $3 \times 3 ， 5 \times 5$; 然后对池化后的结果使用 $1 \times 1$ 的卷 积; 接着对 3 幅特征图进行双线性插值上采样得到 与原特征图相同的大小, 并将 3 组特征与原特征图 进行维度拼接，此时，融合后的特征图维度为原图 的 4 倍; 最后通过一个 $3 \times 3$ 的卷积核将通道数降为 初始大小. 在图 1 的总体网络结构图中可以看出 TGM 在网络中的位置, 而通过上述步骤获取顶层 特征后还需要以不同的倍数上采样并与浅层特征 融合，以参与全局信息的引导

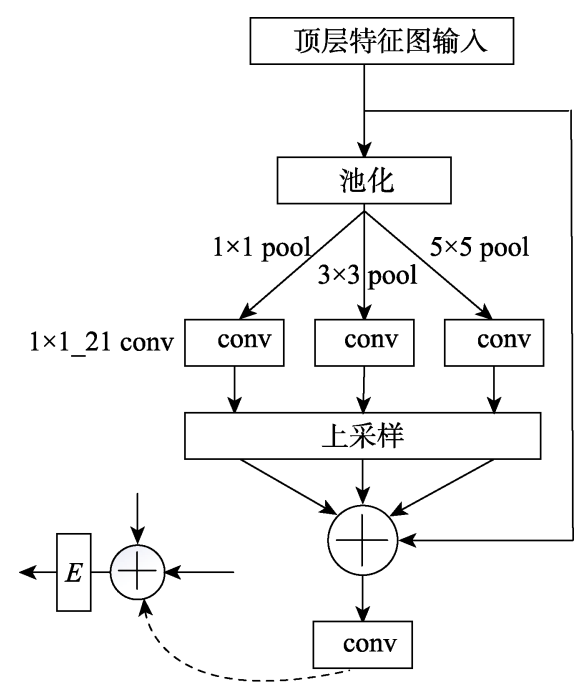

图 3 TGM 具体网络结构

此外，图 3 中融合后的特征图还需经过整合模 块 $E$, 用于融合后的信息过滤. 对于引导模块, 从 主干网络最后得到的特征图经过不同尺度的池化 和合并后, 由于需要高倍数的上采样才能与低层 次的特征图融合, 因此当高倍数的上采样图与原 图直接叠加时会对特征图的完整性和准确性产生 影响, 也就是混叠效应. 为了解决这一问题, 本文 通过整合模块 $E$, 将特征以 2, 4, 6 的倍数平均池化; 经过一个 $3 \times 3$ 卷积后, 再以 $2,4,6$ 的倍数上采样回 原图, 最终将它们相加融合在一起. 因为单个高倍 数的上采样容易失真, 所以通过下采样、池化再上 采样的方式可以减少失真. 另外，整合模块不仅降 低了持续上采样导致的混叠效应，同时还放大了 整个网络的感受野.

\section{5 损失函数}

由于图像的边缘和非边缘分布极度不对称, 且大部分像素点属于非边缘, 如果直接使用交叉 损失函数将无法训练网络. 受 $\mathrm{Liu}$ 等 ${ }^{[12]}$ 的启发, 本
文对损失函数进行了设计. 对边缘真值图进行处 理后，将不同标图人员的标注结果生成边缘概率 图, 边缘概率高于 $\eta$ 的像素点归为正样本, 边缘概 率等于 0 的像素点归为负样本; 因为部分概率低于 $\eta$ 的像素点可能在语义上存在争议, 所以将其舍 弃. 本文设计的损失函数公式为

$$
L\left(X_{i} ; W\right)= \begin{cases}\alpha \cdot \log \left(1-P\left(X_{i} ; W\right)\right), & y_{i}=0 \\ \beta \cdot \log \left(P\left(X_{i} ; W\right)\right), & y_{i}>\eta . \\ 0, & \text { 其他 }\end{cases}
$$

其中, $\alpha=\lambda \cdot \frac{\left|Y^{+}\right|}{\left|Y^{+}\right|+\left|Y^{-}\right|}$和 $\beta=\frac{\left|Y^{-}\right|}{\left|Y^{+}\right|+\left|Y^{-}\right|}$分别表示加 权交叉损失函数的 2 个正负权重; $Y^{+}$和 $Y^{-}$分别表 示正样本集和负样本集; $\lambda$ 为超参数，用于平衡正 负样本； $X_{i}$ 表示图像像素点 $i$ 处的激活值; $y_{i}$ 表 示真值图中的边缘概率; $P(X)$ 表示标准的 Sigmoid 函数; $W$ 表示网络结构中学习的所有参 数. 因此, 每个像素点相对于真值图的损失可以表 示为 $L\left(X_{i} ; W\right)$.

为了加强训练效果, 本文在网络结构中对每 个 block 的输出也进行了监督, 并将所有输出融合 后的图像作为最终边缘图. 因此, 本文将损失分为 侧边损失和融合损失. 本文网络总体损失函数为

$$
\begin{aligned}
& L=\omega_{\text {side }} \cdot L_{\text {size }}+\omega_{\text {fuse }} \cdot L_{\text {fuse }}= \\
& \quad \sum_{i=1}^{|I|}\left(\sum_{k=1}^{K} l\left(X_{i}^{(k)} ; W\right)+l\left(X_{i}^{\text {fuse }} ; W\right)\right) .
\end{aligned}
$$

其中, $\omega_{\text {side }}$ 和 $\omega_{\text {fuse }}$ 分别为侧边损失和融合损失的 2 个权重, 用于调节最终的输出结果; $X_{i}^{(k)}$ 为图像 像素点 $i$ 处第 $k$ 个侧边的激活值; $X_{i}^{\text {fuse }}$ 为融合后 图像的激活值; $|I|$ 为图 $I$ 中的像素总数; $K$ 为侧 输出的数量.

\section{2 实 验}

\section{1 数据集}

实验使用 3 个公共的数据集 $\operatorname{BSDS} 500^{[1]}$, NYUDv2 ${ }^{[19]}$ 和 Multicue ${ }^{[20]}$ 评估网络性能.

BSDS500 是一种应用广泛的边缘检测数据集, 其包含 200 幅训练图像、100 幅验证图像以及 200 幅测试图像. 每幅图像都至少由 5 位标注者手动标 注, 而最后的真值是对所有标注边缘取平均值. 为 防止数据数量不足导致训练过拟合, 实验采用文 献[12]的方法对 BSDS500 数据集进行增强，通过 
对图像的旋转、缩放和随机翻转处理, 将数据集扩 展至 42346 幅. 此外, 实验进一步采用 PASCAL VOC 上下文数据集 ${ }^{[21]}$ 作为训练集的补充, 通过混合 PASCAL VOC 与 BSDS500 数据集, 加强训练效果.

NYUDv2 数据集是由 1449 幅大小为 $640 \times 480$ 像素的图像组成, 分为 381 幅训练图像、414 幅验 证图像和 654 幅测试图像, 该数据集在原图的基础 上提供了对应的 RGB 图像和 HHA 图像, 其中 HHA 图像包含了深度信息. Gupta 等 ${ }^{[22]}$ 将深度图像编码 为 3 种不同的通道: 水平差异、对地高度和表面法 向量的角度. 为防止过拟合, 实验对该数据集进 行了增强，将训练集和对应的真值图以 4 个不同的 角度 $0^{\circ}, 90^{\circ}, 180^{\circ}, 270^{\circ}$ 进行旋转, 再进行左右翻转 处理.

Multicue 数据集是由自然场景的双目视频序 列组成的丰富视频数据集, 该数据集包含 100 个场 景，每个场景都包含一个简短的(10帧左右)视图颜 色序列. 其中，每个场景的左视图中的最后一帧图 像被手动标注成 2 幅真值图, 分别为对象级边缘真 值图和低级轮廓真值图. 实验将 100 幅图像划分为 80 幅训练集和 20 幅测试集，用同样的方法对数据 集进行增强后，对数据集进行训练.

\section{2 实验细节与指标评估}

实验运行的软件环境为 64 位 Ubuntu16.04 系 统, 运行框架为 Pytorch1.3.0 和 MatlabR2016a. 硬 件环境为 Intel(R) Xeon(R) E5-2678CPU @ $2.50 \mathrm{GHz}$, 内存为 $16 \mathrm{~GB}, \mathrm{GPU}$ 为 GeForce GTX TITAN Xp，显存为 $12 \mathrm{~GB}$.

首先，在 ImageNet ${ }^{[23]}$ 上预先训练 VGG16 模 型, 以初始化网络结构, 提高预测结果的准确性. 计算损失时, 实验设置 BSDS500 数据集边缘概率 阈值 $\eta=0.3$ ，损失函数超参数 $\lambda=1.1$; 设 Multicue 边缘数据集 $\eta=0.4, \lambda=1.2 ; \mathrm{NYUD} 2 \mathrm{v}$ 数据集只有 一个真实边缘, 无须设置 $\eta$, 设 $\lambda=1.1$, 侧输出的 损失权重 $\omega_{\text {side }}=0.6$, 融合损失权重 $\omega_{\text {fuse }}=1.0$. 另外, 实验采用随机梯度下降法 (stochastic gradient descent, SGD) 作为网络的优化器, 并将 SGD 的 momentum 参数设为 0.9 , 训练批次大小设为 5 , 初 始学习率设为 $10^{-6}$, 权重衰减值设为 $2 \times 10^{-4}$, 每迭 代 10000 次后学习率下降 0.1 , 总共迭代次数为 40000 次.

对于边缘的评估，实验参照文献[12]，在评估 前执行标准的 NMS 生成最终的边缘映射. 由于修 改 NMS 参数会生成不同的边缘细化结果, 为了比 较真实的网络性能, 需要消除后处理产生的误差.
本文对 Matlab 中 edgesNms 函数进行了参数统一, NMS 的部位参数设为 2 , 抑制边界宽度参数设为 5 , 边缘强度参数设为 1.01 . 此外, 本文采用 3 个广 泛使用的评估指标：数据集尺度上最优 (optimal dataset scale, ODS)、图片尺度上最优(optimal image scale, OIS)、平均精度(average precision, AP). 其中, ODS 表示测试集图像在同一阈值下的计算结果; OIS 表示在测试集的样本中取得最佳阈值后的结 果; AP 为精确率和召回率的曲线面积. 各评估指 标分别定义为

$$
\begin{gathered}
\text { ODS }=\max \left(\frac{2 P_{t} \times R_{t}}{P_{t}+R_{t}}\right) ; \\
\text { OIS }=\frac{1}{N} \times \sum_{i}^{N} \max \left(\frac{2 P_{t}^{i} \times R_{t}^{i}}{P_{t}^{i}+R_{t}^{i}}\right) .
\end{gathered}
$$

其中, $P_{t}$ 和 $R_{t}$ 分别为固定國值为 $t$ 时的精度和查 全率; $N$ 为图像总数; $P_{t}^{i}$ 和 $R_{t}^{i}$ 分别为第 $i$ 幅图像 的 $P_{t}$ 和 $R_{t}$. 精确率和召回率分别为

$$
\begin{aligned}
& P=\frac{\mathrm{TP}}{\mathrm{TP}+\mathrm{FP}} ; \\
& R=\frac{\mathrm{TP}}{\mathrm{TP}+\mathrm{FN}} .
\end{aligned}
$$

$P$ 由正确检测的正样本 (true positive, TP) 和错误检 测的正样本(false positive, FP)计算得到; $R$ 由错误检 测的负样本(false negative, FN)和 TP 计算. 本文使 用综合评价指标 $F_{\text {measure }}$ 具体评估 ODS 和 OIS 的数 值, 定义为 $F_{\text {measure }}=(2 P \times R) /(P+R)$.

\section{3 实验结果分析}

\subsection{1 本文网络模型测试}

为了验证本文提出的网络模型的有效性，实 验对 UNet++结构、FEM 以及 TGM 进行了性能测 试. 首先, 实验在原网络的基础上替换或删除部分 结构. 之后, 将改动后的网络在原始的 BSDS500 数据集上测试评估, 结果如表 1 所示.

表 1 本文网络各模块在 BSDS500 数据集上的性能

\begin{tabular}{lcc}
\hline \multicolumn{1}{c}{ 方法 } & ODS & OIS \\
\hline 本文网络-w-U-w/o-TGM -w/o-FEM & 0.795 & 0.811 \\
本文网络-w-U-w/o-TGM & 0.797 & 0.814 \\
本文网络-w-U & 0.800 & 0.817 \\
本文网络 & 0.803 & 0.821 \\
\hline
\end{tabular}

表 1 中, “-w-U”表示将 UNet++替换成传统的 4 层深度的 U-Net，即去掉中间部分所有 temp 模块 和长短跳跃连接; “-w/o-TGM”表示删除网络中的 TGM; “-w/o-FEM”表示删除网络中的 FEM. 从表 1 
中数据可知, 本文提出的 3 个结构都在一定程度上 提高了边缘检测效果, 原始的 VGG16 外加 U-Net 结构在边缘检测上 ODS 仅为 0.795 , OIS 也只有 0.811. 当添加了 FEM 后, 边缘检测性能开始出现 提升, ODS 上升了 0.002 , OIS 上升了 0.003 , 这说明 了 FEM 的有效性. 在此基础上添加 TGM 后, ODS 和 OIS 分别达到了 0.800 和 0.817 , 而这个指标已 经超越现有 U 型结构的方法. 最后将传统的 U-Net 替换为 UNet++, 总体性能又有了进一步的提升. 本文最终网络在原始 BSDS500数据集上的 ODS 为 0.803 , OIS 为 0.821 , 比最初的模型分别提高了 $1.0 \%$ 和 $1.2 \%$.

\subsection{2 与现有边缘检测网络的比较}

在 BSDS500 数据集上, 实验将本文网络与现有 的基于深度学习的一些网络进行比较, 包括 $\mathrm{LPCB}^{[24]}, \mathrm{CED}^{[25]}$, DeepBoundary ${ }^{[26]}, \mathrm{RCF}^{[12]}, \mathrm{COB}^{[27]}$, $\mathrm{HED}^{[11]}$, DeepContour ${ }^{[28]}, \mathrm{PMI}^{[29]}$. 图 4 所示为不同 网络在 BSDS500 数据集上的评估曲线图. 表 2 所 示为各网络在 BSDS500 数据集上的客观评价结果 统计. 其中, “(pd)”表示在 BSDS500 数据集中额外 添加 PASCAL VOC 数据集进行训练; “ “(pd+ms)”表 示在扩展训练数据集的基础上，在测试过程中将 多尺度图像输人并融合后获得的结果. 从表 2 和图 4 的结果中可以看出, 人类感知在边缘检测中的 ODS 和 OIS 均为 0.803 , 且数据增强和多尺度输人 均能大大提高边缘检测的效果. 本文网络在单一 的 BSDS500 数据集上就取得了很好的效果, 其中 ODS 和 OIS 分别达到了 0.803 和 0.821 , 已经超过 了人类感知. 在添加 PASCAL VOC 数据集后, 本 文网络的性能依然在领先位置, ODS 已经提高到 了 0.815. 而在 PASCAL VOC 数据增强和多尺度输 人下, ODS, OIS 和 AP 分别达到了 0.821，0.839 和 0.869 , 整体上好于现有的基于深度学习的方法,

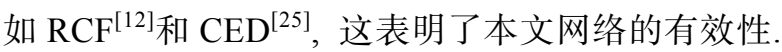

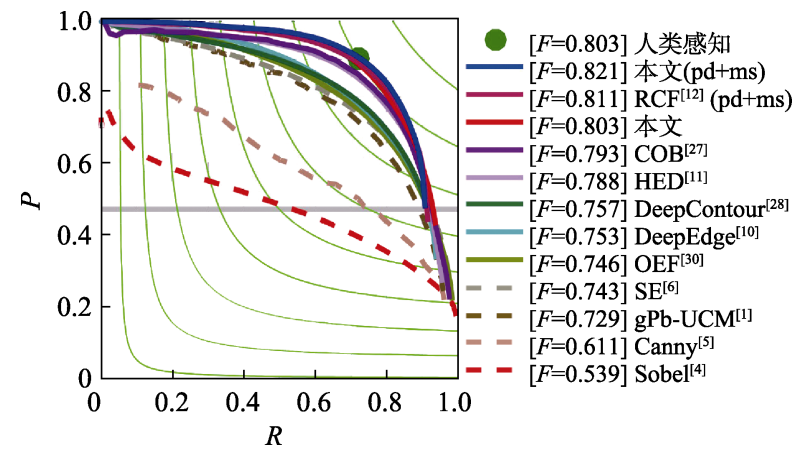

图 4 不同网络在 BSDS500 数据集上的评估曲线
表 2 不同网络在 BSDS500 数据集上的客观指标对比

\begin{tabular}{lccc}
\hline \multicolumn{1}{c}{ 网络 } & ODS & OIS & AP \\
\hline 人类感知 & 0.803 & 0.803 & \\
$\mathrm{PMI}^{[29]}$ & 0.741 & 0.769 & 0.799 \\
DeepContour ${ }^{[28]}$ & 0.757 & 0.776 & 0.800 \\
$\mathrm{HED}^{[11]}$ & 0.788 & 0.808 & 0.840 \\
$\mathrm{COB}^{[27]}$ & 0.793 & 0.820 & 0.859 \\
$\mathrm{RCF}^{[12]}$ & 0.798 & 0.815 & \\
$\mathrm{RCF}^{[12]}(\mathrm{pd})$ & 0.806 & 0.823 & \\
$\mathrm{RCF}^{[12]}(\mathrm{pd}+\mathrm{ms})$ & 0.811 & 0.830 & \\
$\mathrm{DeepBoundary}^{[26]}$ & 0.789 & 0.811 & 0.789 \\
$\mathrm{CED}^{[25]}$ & 0.794 & 0.811 & 0.847 \\
$\mathrm{CED}^{[25]}(\mathrm{pd}+\mathrm{ms})$ & 0.815 & 0.833 & 0.889 \\
$\mathrm{LPCB}^{[24]}$ & 0.800 & 0.816 & \\
$\mathrm{LPCB}^{[24]}(\mathrm{pd})$ & 0.808 & 0.824 & \\
$\mathrm{LPCB}^{[24]}(\mathrm{pd}+\mathrm{ms})$ & 0.815 & 0.834 & \\
本文 & 0.803 & 0.821 & 0.824 \\
本文(pd) & 0.815 & 0.831 & 0.850 \\
本文(pd+ms) & 0.821 & 0.839 & 0.869 \\
\hline
\end{tabular}

图 5 所示为本文网络与其他网络在单一的 BSDS500 数据集上的训练结果主观对比图, 可以看 出, 本文网络的训练结果更接近真值图, 边缘细节 还原度高，且更加清晰. 从整体上看，图像噪声少， 分辨率高。

NYUDv2 数据集有 3 种类型的输人，即 RGB， HHA 和 RGB-HHA. 其中, RGB-HHA 表示对 RGB 和 HHA 的边缘检测结果取平均值. 在实验数据预 处理后, 本文再对 3 种类型的数据集进行了实验. 表 3 所示为不同边缘检测网络在 NYUDv2 数据集 上的客观指标对比，包括 $\mathrm{gPb}-\mathrm{UCM}^{[1]}, \mathrm{gPb}+\mathrm{NG}^{[22]}$, $\mathrm{OEF}^{[30]} ， \mathrm{SE}^{[6]} ， \mathrm{SE}+\mathrm{NG}^{[31]} ， \mathrm{HED}^{[11]} ， \mathrm{RCF}^{[12]}$ 和 $\mathrm{LPCB}^{[24]}$. 图 6 所示为本文网络与其他网络的评估 曲线, 其中所有的结果都是基于单尺度输人的.

从表 3 和图 6 中可以看出, 本文网络在该数据 集上的客观指标均优于其他方法. 在 RGB 上, 本 文网络的优势最为明显, ODS 和 OIS 分别要比 LPCB 高 0.006 和 0.004 . 在 HHA 上, 由于边缘检 测对深度图像的敏感度较差, 包括本文网络在内 的所有方法的客观指标都较低且提升较慢, 本文 网络的 ODS 和 OIS 大致和 LPCB 相同. RGB-HHA 上的 ODS 和 OIS 分别领先 0.002. 虽然 HHA 上的 边缘效果要比 RGB 类型数据差得多, 但平均后的 效果反而比两者都高，可以看出不同类型的特征 

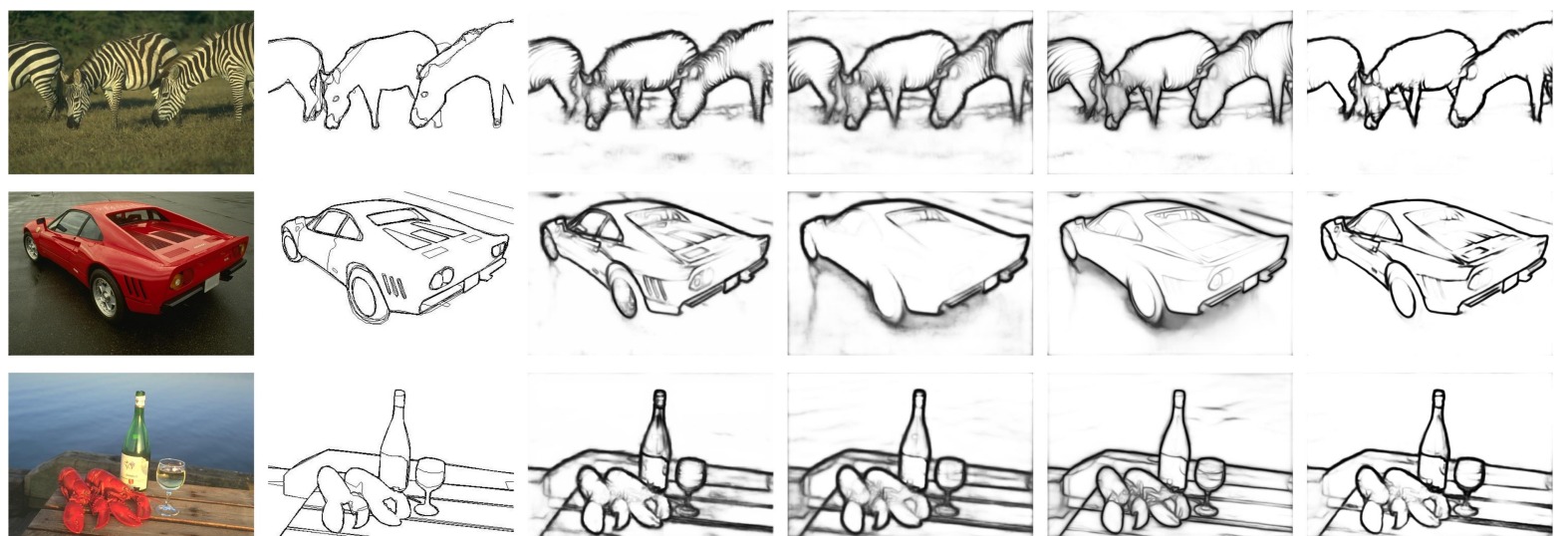

c. $\operatorname{HED}^{[11]}$
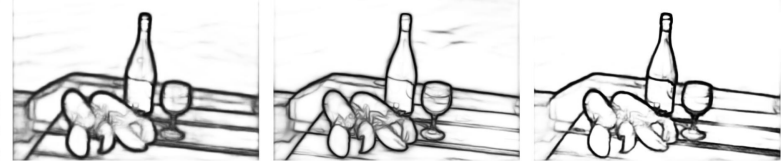

a. 原图

b. 真值图

d. $\mathrm{RCF}^{[12]}$

e. $\mathrm{CED}^{[25]}$

f. 本文

图 5 不同网络在 BSDS500 测试集上的主观对比

表 3 不同网络在 NYUDv2 数据集上的客观指标对比

\begin{tabular}{|c|c|c|c|}
\hline 网络 & ODS & OIS & $\mathrm{AP}$ \\
\hline $\mathrm{gPb}-\mathrm{UCM}^{[1]}(\mathrm{RGB})$ & 0.632 & 0.661 & 0.562 \\
\hline $\mathrm{gPb}+\mathrm{NG}^{[22]}(\mathrm{RGB})$ & 0.687 & 0.716 & 0.629 \\
\hline $\mathrm{OEF}^{[30]}(\mathrm{RGB})$ & 0.651 & 0.667 & \\
\hline $\mathrm{SE}^{[6]}(\mathrm{RGB})$ & 0.695 & 0.708 & 0.679 \\
\hline $\mathrm{SE}+\mathrm{NG}^{[31]}(\mathrm{RGB})$ & 0.706 & 0.734 & 0.738 \\
\hline $\mathrm{HED}^{[11]}$ (RGB) & 0.720 & 0.734 & 0.734 \\
\hline $\operatorname{HED}^{[11]}(\mathrm{HHA})$ & 0.682 & 0.695 & 0.702 \\
\hline $\mathrm{HED}^{[11]}(\mathrm{RGB}-\mathrm{HHA})$ & 0.746 & 0.761 & 0.786 \\
\hline $\mathrm{RCF}^{[12]}(\mathrm{RGB})$ & 0.729 & 0.742 & \\
\hline $\mathrm{RCF}^{[12]}(\mathrm{HHA})$ & 0.705 & 0.715 & \\
\hline $\mathrm{RCF}^{[12]}(\mathrm{RGB}-\mathrm{HHA})$ & 0.757 & 0.771 & \\
\hline $\mathrm{LPCB}^{[24]}(\mathrm{RGB})$ & 0.739 & 0.754 & \\
\hline $\mathrm{LPCB}^{[24]}(\mathrm{HHA})$ & 0.707 & 0.719 & \\
\hline $\mathrm{LPCB}^{[24]}(\mathrm{RGB}-\mathrm{HHA})$ & 0.762 & 0.778 & \\
\hline 本文(RGB) & 0.745 & 0.758 & 0.760 \\
\hline 本文(HHA) & 0.707 & 0.718 & 0.729 \\
\hline 本文(RGB-HHA) & 0.764 & 0.780 & 0.791 \\
\hline
\end{tabular}

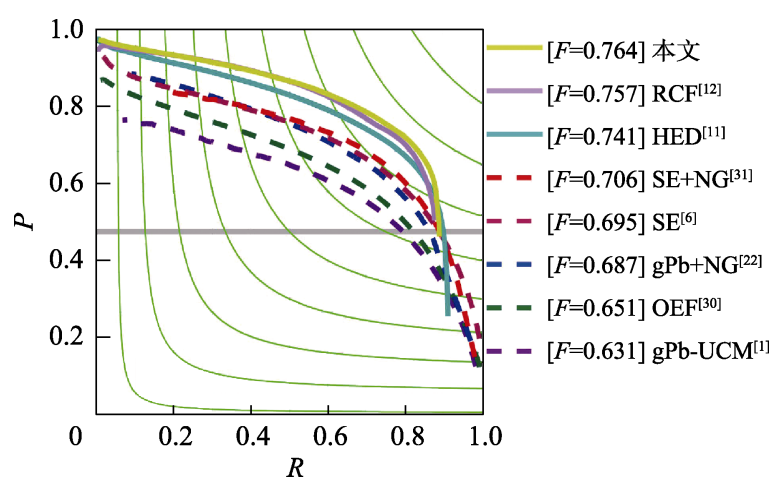

图 6 不同网络在 NYUDv2 数据集上的评估曲线

对边缘检测效果的提升很有帮助. 最终的主观效 果如图 7 所示.

最后, 本文在 Multicue 数据集上进行了测试.

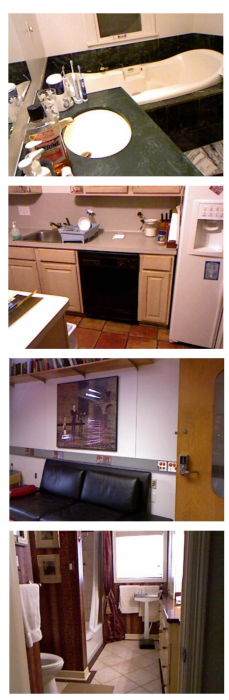

a. 原图

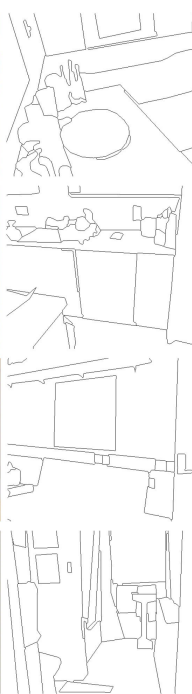

b. 真值图

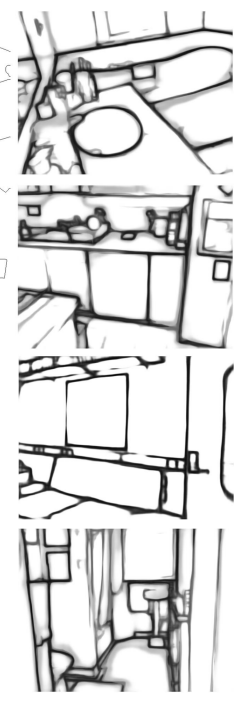

c. 效果图
图 7 本文网络在 NYUDv2 数据集上的检测结果

该数据集由 2 个子数据集组成, 即 Multicue 边界和 Multicue 边缘. 本文网络在这 2 个子数据集上都进 行了训练, 同样取得了较好的边缘效果, 主观效果 如图 8 所示.

虽然本文网络在上述测试中整体效果较好, 但在部分图像上的效果并不令人满意, 如图 9 所 示. 实验同样对 $\mathrm{HED}^{[11]}$ 和 $\mathrm{RCF}^{[12]}$ 算法进行了测试, 在 3 幅图像上出现了类似的错误，即网络对不同尺 度边缘的特征融合出现了错误, 可以看出第 1 幅图 像缺失了脸部特征, 但背景的细节边缘过于明显, 而后 2 幅图像背景边缘过于复杂和模糊. 虽然基于 深度学习的边缘检测技术比传统的算法在性能上 有很大提升, 对光照和阴影等影响因素的鲁棒性 也有所增强, 但是对背景过于复杂的边缘图像依 然存在检测失败问题. 造成上述问题的原因由 2 部 分组成：首先，由于网络对不同尺度边缘的特征融 


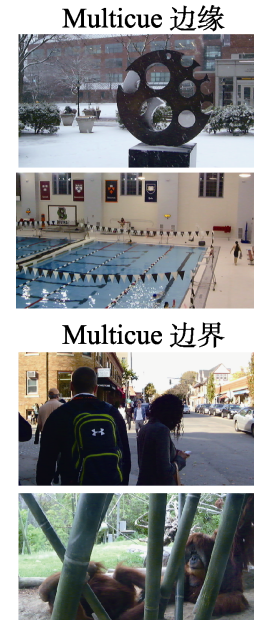

a. 原图
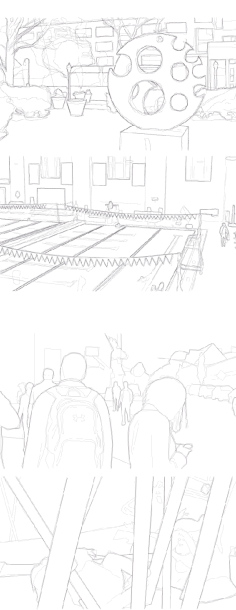

b. 真值图
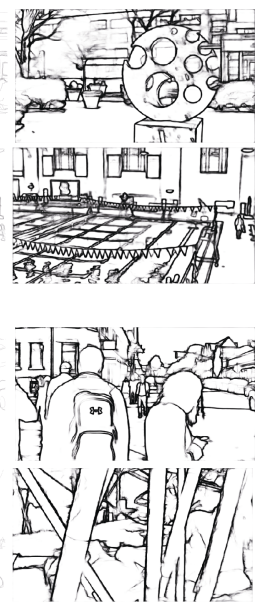

c. 效果图
图 8 本文网络在 Multicue 数据集上的检测结果

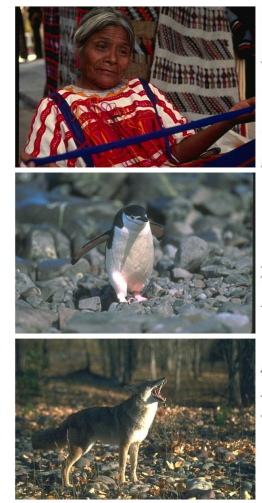

a. 原图

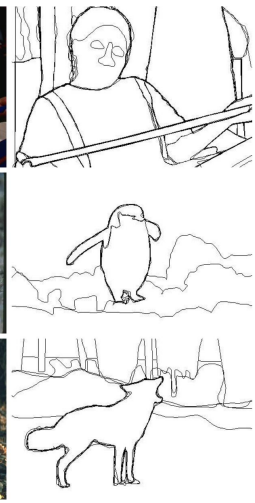

b. 真值图

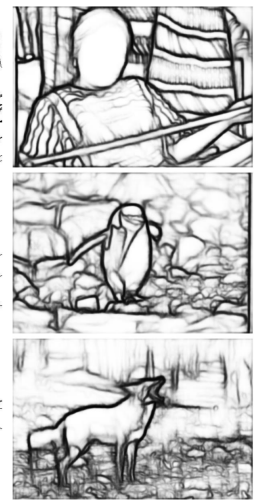

c. 效果图
图 9 本文网络在 BSDS500 数据集上失败案例

合出现了错误，导致部分图像缺失了一些边缘特 征而背景杂质却有很多, 而图像的边缘特征本身 就存在尺度不统一的情况, 因此网络在特征融合 时需要对低级特征进行更精细的区分，以过滤掉 非显著区域的小尺度边缘，防止在复杂背景下边 缘的杂乱; 其次, BSDS500 数据集也存在一些问 题, 早期的数据集数量较少, 且不同标图人员对不 同图像的边缘认知并不是完全统一的, 容易造成 边缘信息的圥余或缺失, 使训练困难. 另外, 本文 网络也存在着一些小缺陷, 过多的下采样使输出 的边缘线条粗䇿且模糊，虽然可以通过后处理进 行边缘细化, 但这也是一个值得进一步探索和解 决的问题.

\section{3 结 语}

为提高复杂自然场景下边缘检测的性能，本 文提出了多深度特征增强与顶层信息引导的边缘
检测网络. 该网络采用 UNet++结构作为网络主干, 增强多深度特征的提取能力; 在下采样中设计了 尺度增强模块, 增强多尺度特征; 在上采样中设计 了顶层信息引导模块, 增强边缘的高层语义信息. 实验结果表明, 本文网络与现有的边缘检测网络 相比, 在 BSDS500, NYUDv2 和 Multicue 3 个数据 集上均取得了更好的效果, 其中, 在 BSDS500 数 据集上的 ODS, OIS, AP 分别达到了 0.821，0.839, 0.869 ; 同时, 在主观视觉效果上, 网络的边缘检 测结果更接近真值, 多尺度表现效果更佳. 虽然本 文网络在客观指标上优于其他方法, 但是仍存在 边缘过粗、背景复杂部分检测效果一般等问题. 后 续工作将围绕这些问题继续展开研究, 进一步提 高边缘检测的性能.

\section{参考文献(References):}

[1] Arbeláez P, Maire M, Fowlkes C, et al. Contour detection and hierarchical image segmentation[J]. IEEE Transactions on Pattern Analysis and Machine Intelligence, 2011, 33(5): 898-916

[2] Ferrari V, Fevrier L, Jurie F, et al. Groups of adjacent contour segments for object detection[J]. IEEE Transactions on Pattern Analysis and Machine Intelligence, 2008, 30(1): 36-51

[3] Girshick R, Donahue J, Darrell T, et al. Rich feature hierarchies for accurate object detection and semantic segmentation[C] //Proceedings of the IEEE Conference on Computer Vision and Pattern Recognition. Los Alamitos: IEEE Computer Society Press, 2014: 580-587

[4] Sobel I E. Camera models and machine perception[M]. Stanford: Stanford University, 1970

[5] Canny J. A computational approach to edge detection[J]. IEEE Transactions on Pattern Analysis and Machine Intelligence, 1986, 8(6): 679-698

[6] Dollár P, Zitnick C L. Fast edge detection using structured forests[J]. IEEE Transactions on Pattern Analysis and Machine Intelligence, 2015, 37(8): 1558-1570

[7] Konishi S, Yuille A L, Coughlan J M, et al. Statistical edge detection: learning and evaluating edge cues[J]. IEEE Transactions on Pattern Analysis and Machine Intelligence, 2003, 25(1): 57-74

[8] Martin D R, Fowlkes C C, Malik J. Learning to detect natural Image boundaries using local brightness, color, and texture cues[J]. IEEE Transactions on Pattern Analysis and Machine Intelligence, 2004, 26(5): 530-549

[9] Ganin Y, Lempitsky V. $N^{4}$-Fields: neural network nearest neighbor fields for image transforms[C] //Proceedings of Asian Conference on Computer Vision. Heidelberg: Springer, 2014: 536-551

[10] Bertasius G, Shi J B, Torresani L. DeepEdge: a multi-scale bifurcated deep network for top-down contour detection[C] //Proceedings of the IEEE Conference on Computer Vision and Pattern Recognition. Los Alamitos: IEEE Computer Society 
Press, 2015: 4380-4389

[11] Xie S N, Tu Z W. Holistically-nested edge detection[C] //Proceedings of the IEEE International Conference on Computer Vision. Los Alamitos: IEEE Computer Society Press, 2015: 1395-1403

[12] Liu Y, Cheng M M, Hu X W, et al. Richer convolutional features for edge detection[C] //Proceedings of the IEEE Conference on Computer Vision and Pattern Recognition. Los Alamitos: IEEE Computer Society Press, 2017: 5872-5881

[13] He J Z, Zhang S L, Yang M, et al. Bi-directional cascade network for perceptual edge detection[C] //Proceedings of the IEEE Conference on Computer Vision and Pattern Recognition. Los Alamitos: IEEE Computer Society Press, 2019: 3823-3832

[14] Simonyan K, Zisserman A. Very deep convolutional networks for large-scale image recognition[OL]. [2020-11-02]. https:// arxiv.org/abs/1409.1556

[15] He K M, Zhang X Y, Ren S Q, et al. Deep residual learning for image recognition $[\mathrm{C}] / /$ Proceedings of the IEEE Conference on Computer Vision and Pattern Recognition. Los Alamitos: IEEE Computer Society Press, 2016: 770-778

[16] Ronneberger O, Fischer P, Brox T. U-Net: convolutional networks for biomedical image segmentation[C] //Proceedings of the 18th International Conference on Medical Image Computing and Computer-Assisted Intervention. Heidelberg: Springer, 2015: 234-241

[17] Zhang Z L, Zhang X Y, Peng C, et al. ExFuse: enhancing feature fusion for semantic segmentation[C] //Proceedings of the 15th European Conference on Computer Vision. Heidelberg: Springer, 2018: 273-288

[18] Chen L C, Papandreou G, Kokkinos I, et al. DeepLab: semantic image segmentation with deep convolutional nets, atrous convolution, and fully connected CRFs[J]. IEEE Transactions on Pattern Analysis and Machine Intelligence, 2018, 40(4): $834-848$

[19] Silberman N, Hoiem D, Kohli P, et al. Indoor segmentation and support inference from RGBD images[C] //Proceedings of the 12th European Conference on Computer Vision. Heidelberg: Springer, 2012: 746-760

[20] Mely D A, Kim J, McGill M, et al. A systematic comparison between visual cues for boundary detection[J]. Vision Research, 2016, 120(5): 93-107

[21] Mottaghi R, Chen X J, Liu X B, et al. The role of context for object detection and semantic segmentation in the wild $[\mathrm{C}]$
//Proceedings of the IEEE Conference on Computer Vision and Pattern Recognition. Los Alamitos: IEEE Computer Society Press, 2014: 891-898

[22] Gupta S, Arbeláez P, Malik J. Perceptual organization and recognition of indoor scenes from RGB-D images[C] //Proceedings of the IEEE Conference on Computer Vision and Pattern Recognition. Los Alamitos: IEEE Computer Society Press, 2013: 564-571

[23] Deng J, Dong W, Socher R, et al. ImageNet: a large-scale hierarchical image database[C] //Proceedings of the IEEE Conference on Computer Vision and Pattern Recognition. Los Alamitos: IEEE Computer Society Press, 2009: 248-255

[24] Deng R X, Shen C H, Liu S J, et al. Learning to predict crisp boundaries[C] //Proceedings of the 15th European Conference on Computer Vision. Heidelberg: Springer, 2018: 570-586

[25] Wang Y P, Zhao X, Huang K Q. Deep crisp boundaries[C] //Proceedings of the IEEE Conference on Computer Vision and Pattern Recognition. Los Alamitos: IEEE Computer Society Press, 2017: 1724-1732

[26] Kokkinos I. Pushing the boundaries of boundary detection using deep learning[OL]. [2020-11-02]. https://arxiv.org/abs/ 1511. 07386

[27] Maninis K K, Pont-Tuset J, Arbeláez P, et al. Convolutional oriented boundaries[C] //Proceedings of the 14th European Conference on Computer Vision. Heidelberg: Springer, 2016: 580-596

[28] Shen W, Wang X G, Wang Y, et al. DeepContour: a deep convolutional feature learned by positive-sharing loss for contour detection[C] //Proceedings of the IEEE Conference on Computer Vision and Pattern Recognition. Los Alamitos: IEEE Computer Society Press, 2015: 3982-3991

[29] Isola P, Zoran D, Krishnan D, et al. Crisp boundary detection using pointwise mutual information[C] //Proceedings of the 13th European Conference on Computer Vision. Heidelberg: Springer, 2014: 799-814

[30] Hallman S, Fowlkes C C. Oriented edge forests for boundary detection[C] //Proceedings of the IEEE Computer Society Conference on Computer Vision and Pattern Recognition. Los Alamitos: IEEE Computer Society Press, 2015: 1732-1740

[31] Gupta S, Girshick R, Arbeláez P, et al. Learning rich features from RGB-D images for object detection and segmentation[C] //Proceedings of the 13th European Conference on Computer Vision. Heidelberg: Springer, 2014: 345-360 